\title{
Effects of disorder on high-temperature superconductors
}

\author{
Tai Kai $\mathrm{Ng}$ \\ Department of Physics, Hong Kong University of Science and Technology, Kowloon, Hong Kong \\ and AT\&T Bell Laboratories, Murray Hill, New Jersey 07974
}

(Received 13 November 1991)

\begin{abstract}
The effect of disorder on high-temperature superconductors is studied using a phenomenologically modified scaling theory for disordered interacting fermions. It is found that the effect of disorder on high-temperature superconductors is very different from the effect on a normal metal because of the "marginal"-Fermi-liquid effect. In particular, we found that the system always scales toward strong disorder if the "marginal" effect is strong enough. Correspondingly, the magnetic susceptibility goes to zero.
\end{abstract}

With the discovery of high- $T_{c}$ superconductors, ${ }^{1}$ the problem of highly correlated electronic systems becomes one of the most active areas in condensed-matter physics. Aside from the numerous observed anomalous "clean metal" normal-state properties, ${ }^{2}$ the effect of disorder on the high- $T_{c}$ superconductors is also highly unusual. Unlike normal metals where in the limit of weak disorder there exists a so-called weak-localization regime in which logarithmic corrections to properties like conductance $\sigma$ and density of state $\rho$ are found ${ }^{3}$ [in two dimensions (2D)], in high- $T_{c}$ superconductors such a regime seems to be missing and it appears that the superconducting state can be destroyed and the system driven directly into an insulating state in the presence of very weak disorder. ${ }^{4.5}$ The purpose of this paper is to show that this unusual behavior of the high- $T_{\text {c }}$ superconductors can be explained from their anomalous (clean) normal-state behavior in the framework of scaling theory for disordered interacting fermions. Moreover, as in normal metals where the localization effect is directly related to the magnetic properties, we find that, corresponding to the strong-localization effect, the magnetic susceptibility goes to zero.

The interplay between electron-electron interaction and Anderson localization is at present understood in terms of a Fermi-liquid framework of diffusing quasiparticles with scale-dependent Fermi-liquid parameters. ${ }^{6}$ The scale dependence of these parameters is determined by a set of renormalization-group ( $R G$ ) equations subject to certain initial conditions which are determined by the Fermiliquid parameters of the clean system. However, in the case of high- $T_{\text {c }}$ superconductors, there exists no microscopic theory for the normal state at present and it is not even sure whether the normal state can be described as a Fermi liquid. (For example, in theories 7,8 with spincharge separation, the low-energy excitations do not carry both the spin and charge quantum numbers of an electron and cannot be described as a "Landau"-type quasiparticle.) In view of the lack of a microscopic theory, we shall tackle the problem in a phenomenological way. We observe that many of the properties of the high- $T_{c}$. superconductors can be understood by introducing a phenomenological "marginal"-Fermi-liquid description ${ }^{9,10}$ where the one-particle electron Green's function has an inverse lifetime $\tau^{-1}$ linear in temperature or frequency $\operatorname{Im} \Sigma(T, k$, $\omega) \sim \max (T, \omega)]$ corresponding to a scale-dependent effective mass

$$
m^{*} / m \sim \ln \left(\omega_{c} / x\right), x=\max (\omega, T),
$$

and also with nonsingular spin and charge susceptibilities. We make the phenomenological assumption that the high- $T_{c}$ superconductors can be described by the same set of RG equations as in normal metals in the presence of disorder, except that the "input" Landau parameters are modified corresponding to the marginal-Fermi-liquid phenomenology. We shall see that the behavior of the scaling equations is drastically modified from the normal-metal case as a result of the marginal-Fermi-liquid effect.

We shall begin with a brief review of the scaling theory of disordered interacting electrons. Our discussion will be restricted to $2 \mathrm{D}$ since it is believed that normal-state transport of the high- $T_{c}$ superconductors is essentially $2 \mathrm{D}$ in character. ${ }^{4.5}$

In the Fermi liquid plus scaling description of the disordered-interacting-fermions problem, the low-energy excitations are quasiparticles as in the normal Landau Fermi-liquid theory except ${ }^{6.11}$ (i) the quasiparticles are diffusive, with a quasiparticle diffusion constant $D_{Q}$, but not ballistic as in the normal Fermi-liquid theory, (ii) the Landau interaction parameters $f_{\sigma \sigma^{\prime}}$ do not depend on the angle since momentum is not conserved in a disordered system and the most important events are $s$-wave scattering, and (iii) the Landau interaction function $f_{\sigma \sigma^{\prime}}$, diffusion constant $D_{Q}$ and the quasiparticle density of states $\rho_{Q}$ are all scale dependent. Otherwise, the normal Fermi-liquid phenomenology holds and the compressibility $d n / d \mu$, the linear term in specific heat $\gamma$, spin susceptibility $\chi$, and conductivity $\sigma$, are given by the following expressions: ${ }^{11}$

$$
\begin{aligned}
& (d n / d \mu) / \rho_{0}=\left(\rho_{Q} / \rho_{0}\right)\left(1-2 \gamma_{S}\right) \\
& \gamma / \gamma_{0}=\rho_{Q} / \rho_{0}=Z \\
& \chi / \chi_{0}=\left(\rho_{Q} / \rho_{0}\right)\left(1-2 \gamma_{t}\right), \\
& \sigma=(d n / d \mu) D
\end{aligned}
$$

where

$$
D=D_{Q} /\left(1-2 \gamma_{S}\right),
$$

and $\rho_{0}, \gamma_{0}, \chi_{0}$ are the corresponding quantities for the 
noninteracting system. Other quantities like the spin diffusion constant $D_{S}=D_{Q} /\left(\chi / \chi_{0}\right)$, thermal conductivity, etc., can be obtained in a similar fashion. ${ }^{6,11}$

One can also introduce the single-particle density of states $\rho_{1}(E)=a \rho_{Q}(E)$, which is measured in tunneling experiments. $a$ is the quasiparticle weight which enters into the one-particle Green's function. It is clear that $Z$, $2 \gamma_{S}$, and $2 \gamma_{1}$ play the role of the Landau parameters $m^{*} / m, A_{0}^{S}$, and $A_{0}^{a}$ in the Fermi-liquid theory, whereas $a$ corresponds to the usual quasiparticle renormalization constant. These parameters are governed by a set of RG equations which describe the behavior of these parameters at a scale $q<l^{-1}$ and $\omega<\tau^{-1}$, where $l$ is the elastic scattering mean free path and $\tau$ the corresponding scattering time. The physical meaning of the cutoff is clear. The quasiparticles are diffusive only for the distance scale $q^{-1} \gg l$. For a distance of $q^{-1} \leq 1$, electron motion is ballistic and should be described by a cleanmetal theory. In particular, in the limit when the impurity concentration is low, the cutoff distance $l$ is large and the system would be already in the (clean) Landau Fermi-liquid regime at this scale where the parameters $Z$, $\gamma_{S}, \gamma_{t}$, and $a$ are just the usual Landau Fermi-liquid parameters for the clean system. Moreover, $D_{Q}=v_{F}^{2} \tau / 2$ is determined by the scattering phase shift of a single impurity in the interacting Fermi liquid, 6,11 i.e., the (clean) Landau parameters enter as the initial values of the $R G$ at the scale $q-l^{-1}$.

The RG equations for these "Landau" parameters were first derived by Finkelstein ${ }^{12}$ for the case of electrons interacting via a long-range Coulomb potential and was later generalized to the case of short-range potentials by Castellani and co-workers. ${ }^{11,13}$ Their theories were valid to lowest order in the disorder parameter $t=1 / 4 \pi^{2} \sigma$ which explicitly suppressed the maximally crossed diagrams that give rise to weak localization in 2D noninteracting electrons and can be considered as a scaling theory for the "pure" interaction problem. $11-13$ Notice that by suppressing the maximally crossed diagrams, the interaction effects in the particle-particle channel are also suppressed. Thus the present scaling theory cannot describe, for example, the competition between superconductivity and localization (see also Ref. 14).

With these limitations in mind, we proceed to write down the (lowest order) scaling equations, first for the case with short-range (repulsive) interaction, ${ }^{13}$

$$
\begin{aligned}
& d \gamma_{1} / d \xi=t\left[\left(\gamma_{2}-\gamma_{1}\right)^{2}+\gamma_{2}\right], \\
& d \gamma_{2} / d \xi=t \gamma_{1}\left(1+\gamma_{2}\right) \text {, } \\
& Z\left(1-2 \gamma_{S}\right)=C_{\kappa} \text {, } \\
& d t / d \xi=t^{2}\left(2 \gamma_{S}+\left[\left(1-2 \gamma_{S}\right) / 2 \gamma_{S}\right] \ln \left(1-2 \gamma_{S}\right)\right. \\
& \left.+3\left\{1-\left[\left(1+\gamma_{2}\right) / \gamma_{2}\right] \ln \left(1+\gamma_{2}\right)\right\}\right) \text {, } \\
& d \ln \rho_{1} / d \xi=\frac{1}{2} t\left[\ln \left(1-2 \gamma_{S}\right)+3 \ln \left(1+\gamma_{2}\right)\right] \text {, }
\end{aligned}
$$

where $\gamma_{S}=\gamma_{1}-\frac{1}{2} \gamma_{2}, \gamma_{t}=-\frac{1}{2} \gamma_{2}$, and $\xi=-\ln (T \tau)$. $\gamma_{S}$, $\gamma_{t}$, and $Z$ are the Landau parameters defined in Eqs. $(2 \mathrm{a})-(2 \mathrm{c}), \xi$ is the scale parameter, and $t$ is the disorder parameter. $C_{\kappa}$ is a numerical constant proportional to the compressibility of the clean system. $\rho_{1}$ is the single- particle density of states. Notice that Eq. (3c) together with Eqs. (2a) and (2b) implies that the compressibility of the interacting system is unrenormalized by disorder, as is expected on physical grounds. ${ }^{13}$

In the case with Coulomb interaction, the scaling equations (3a)-(3c) remain the same except $C_{\kappa}=0$, but (3d) and $(3 \mathrm{e})$ are modified to $11-13$

$$
\begin{aligned}
& \frac{d t}{d \xi}=t^{2}\left(4-3 \frac{1+\gamma_{2}}{\gamma_{2}} \ln \left(1+\gamma_{2}\right)\right), \\
& d \ln \rho_{1} / d \xi=-\frac{1}{2} t\left[\xi-3 \ln \left(1+\gamma_{2}\right)\right] .
\end{aligned}
$$

The difference in the Coulomb case can be understood by noting that in the limit $q \rightarrow 0$, the spin singlet scattering amplitude $\gamma_{S}$ (or $\gamma_{1}$ ) is dominated by the screened Coulomb interaction $\Gamma(q, \omega)=v(q) /[1+v(q) \pi(q, \omega)]$ $\rightarrow \pi(q, \omega)^{-1}$ because of the divergence in $v(q)$ as $q \rightarrow 0$, where $\pi(q, \omega)$ is the polarization bubble. ${ }^{15}$ Furthermore, $\pi(q \rightarrow 0,0)$ is just the compressibility which is unrenormalized in the present theory. This "universal" behavior leads to the absence of $\gamma_{S}$ in Eq. (4). Notice also that $C_{K}=0$ in the Coulomb case because of the divergence of $v(q)$ in the density-density response function $\chi_{\mathrm{dd}}=\pi /[1$ $-v(q) \pi] \rightarrow 0$ as $q \rightarrow 0 .^{11-13}$

Now we shall examine how the RG equations (3) and (4) are modified in the phenomenology of the high- $T_{c}$ superconductors. Assuming that $m^{*} / m$ (or $Z$ ) $\sim 1 / a$ is given by Eq. (1) in the clean normal state of the high- $T_{c}$ superconductors (notice that this assumption implies also that the momentum dependence of the one-particle selfenergy gives no singular contribution to $\mathrm{m}^{*} / \mathrm{m}$ ) and that the compressibility and magnetic susceptibility remain finite (no logarithmic correction) as $T \rightarrow 0,{ }^{10}$ one can determine using Eq. (2) the "bare" Landau parameters $Z$, $a, \gamma_{S}, \gamma_{t}$ entering into the RG equations (3) and (4). Notice that instead of being constants, these bare parameters are scale-dependent themselves in the (clean) marginal Fermi liquids. The scale dependence of the Landau parameters can be summarized in the following equations:

$$
\begin{aligned}
& d Z / d \xi=c_{m}, \\
& d \gamma_{1} / d \xi=-c_{m} \gamma_{1} / Z, \\
& d \gamma_{2} / d \xi=-c_{m}\left(1+\gamma_{2}\right) / Z \\
& d t / d \xi=d \ln \rho_{1} / d \xi=0,
\end{aligned}
$$

where $c_{m}>0$ is a phenomenological parameter characterizing the strength of the $\ln \omega_{c} / T$ term in the marginalFermi-liquid phenomenology. Equations (5b) and (5c) implies that there exists logarithmic corrections to the Landau parameters $Z \gamma_{1}$ and $Z \gamma_{2}$ as a result of the marginal-Fermi-liquid effect, as was pointed out in Ref. 10. Notice that the single-particle density of states $\rho_{1} \sim a \rho_{Q}$ is unrenormalized in the (clean) marginal Fermi liquid. Notice also that we have assumed $d t / d \xi=0$. In the original marginal-Fermi-liquid phenomenology of Varma et al., ${ }^{9}$ a vertex correction $\sim\left(\ln \omega_{c} / T\right)^{2}$ was found for the single-impurity Born-scattering amplitude. ${ }^{16}$ Such a behavior was not included in our phenomenological equation (5d) since the scattering phase shift is always bound by the unitarity limit and the $\left(\ln \omega_{c} / T\right)^{2}$ behavior 
should vanish below some critical energy. ${ }^{10,16}$ One may also put back the vertex correction phenomenologically into (5d) with the scattering phase shift saturated at the unitarity limit at low frequency. ${ }^{10}$ The transient behaviors of the $R G$ equations will get modified as a result, but the low-energy behavior and fixed points of the RG equations will not be modified.

To proceed further we shall make the assumption that the $R G$ equation in the presence of disorder is just a sum of Eq. (5) and the disorder contributions (3) and (4) without any further modification. It has to be emphasized that this assumption is purely phenomenological and its validity is unknown in the absence of a microscopic theory. Nevertheless, the physical meaning behind this assumption can be understood roughly as follows: The $\mathrm{RG}$ equations can be derived by considering various Feynman diagram contributions to the parameters $Z, \gamma_{1}, \gamma_{2}$, and $t$. These diagrams can, in general, be divided into two parts. One part contains all diagrams having no impurity scattering corrections, i.e., the terms coming from the clean system. The other part contains the rest of all the diagrams where the electron interacts at least once with the impurity potential. The RG equations are given by the sum of the two contributions. In normal Fermi liquids the first part is zero whereas the second part gives to lowest order in $t$ Eqs. (3) and (4). For marginal Fermi liquids, our phenomenological assumption is that contributions from the disorder-induced part are not modified by the marginal-Fermi-liquid behavior (except for corrections arising implicitly from the modified scaling behavior). Notice that in ordinary Fermi liquids, the scaling equations are dominated by contributions from the diffusion poles of the quasiparticles. ${ }^{13}$ Our phenomenology implies that the same is true in the present case and may be incorrect if, for example, there exist other important low-energy excitations in the system as proposed in the original marginal-Fermi-liquid phenomenology ${ }^{9}$ and their contributions to transport behavior have to be examined.

We shall now study the behaviors of the new marginalFermi-liquid RG equations. First we shall examine briefly the high-temperature corrections to the conductance $\sigma$ and density of states $\rho_{1}$. Afterward we shall study the scaling behavior and fixed points of these new equations.

The high-temperature corrections to $\sigma$ and $\rho_{1}$ can be obtained by integrating the scaling equations (3)-(5), with the parameters $Z, \gamma_{1}, \gamma_{2}$, and $t$ the bare parameters of the clean system. For normal Fermi liquids these parameters are scale independent and the integral can be performed easily. One obtains $\ln (T \tau)$ corrections to $\sigma$ and $\delta \rho_{1} / \rho_{1}$ in the case of short-ranged interactions ${ }^{13}$ and an extra $(\ln T \tau)^{2}$ correction to $\delta \rho_{1} / \rho_{1}$ in the case of Coulomb interaction. ${ }^{11-13}$ For a marginal Fermi liquid, a parallel analysis indicates that extra corrections which cannot be written simply as a $\ln T \tau$ correction are found both for the conductance $\sigma$ and density of states $\delta \rho_{1} / \rho_{1}$. Writing $Z(\xi)=Z_{0}+c_{m} \xi$ and assuming $c_{m} \xi \ll Z_{0}$, we find that the deviation from $\ln T \tau$ behavior comes at order $t\left(c_{m} / Z_{0}\right)$, whereas in normal Fermi liquids, deviation from $\ln T \tau$ behavior comes at order $t^{2}$ (except the correction to $\delta \rho_{1} / \rho_{1}$ in the Coulomb case). In the weak-disorder (small- $t$ ) limit, the deviation from $\ln T \tau$ behavior is dom- inated by the marginal-Fermi-liquid term, and with a magnitude much stronger than the case of normal Fermi liquids. This is in qualitative agreement with experiments where it is difficult to find well-defined $\ln T \tau$ behavior in conductance over an extensive region of temperature for high- $T_{c}$ superconductors. ${ }^{5}$

To study the scaling behavior of the new RG equations, it is useful first to recall the results for normal Fermi liquids. For a normal Fermi liquid, it is well known that the disorder parameter $t$ is reduced upon scaling ${ }^{11-13}$ but the scaling equations diverge at some finite scale $\xi \rightarrow \xi_{c}$ where $\gamma_{2} \sim\left|\xi-\xi_{c}\right|^{-1}$ and $Z \sim\left|\xi-\xi_{c}\right|^{-3}$, with $t$ remaining finite. This is true both for the case of short-ranged repulsive interaction and Coulomb interaction. The divergence of the parameters $\gamma_{2}$ and $Z$ indicates that the RG equations, which are derived under the assumption of weak disorder, is no longer valid as $\xi \rightarrow \xi_{c}{ }^{13}$ Nevertheless, they do follow the qualitative picture that localized spin clusters are formed at low energy and the impurity scattering effect is reduced by spin-triplet scattering. ${ }^{11-13}$ For a marginal Fermi liquid, notice that if $\gamma_{i} / Z \rightarrow 0(i$ $=1,2$ ) and $Z \rightarrow \infty$ upon scaling, the RG equations for $\gamma_{1}$ and $\gamma_{2}$ become identical to the ones for normal Fermi liquid, and the same fixed point will be found. Thus the fixed point for a normal Fermi liquid is also a fixed point in the present case. However, we find another stable fixed point in the case of short-ranged interaction for a marginal Fermi liquid. This new fixed point corresponds to $\gamma_{2}^{*} \rightarrow-1$ as $\xi \rightarrow \infty$. Substituting $\gamma_{2}^{*}=-1$ into the sums of Eqs. (3) and (5), we find $\gamma_{1}^{*}=-2 t^{*} /\left(t^{*}+2 c_{m}\right)$ where $d t^{*} / d \xi=0$. Solving the equations numerically we find $t^{*} \sim 3.258 c_{m}$ and also $\rho_{1}^{*} \rightarrow 0$.

To understand the role of the two fixed points we fix the initial values of $\gamma_{1}, \gamma_{2}, c_{m}$, and $C_{\kappa}$ and look at the scaling behavior of the equations as a function of initial value $t$. For $t$ less than a critical value $t_{c} \sim c_{m}$ ( $t_{c}$ depends on the initial values of all parameters in general), we find that the RG equations scale to the new fixed point $\gamma_{2}^{*} \rightarrow-1$, whereas for $t>t_{c}$, they scale to the normal Fermi-liquid fixed point $\gamma_{2}^{*} \rightarrow \infty$. Thus the new fixed point [which we shall call the marginal-Fermi-liquid fixed point (MFFP)] is only stable for weak disorder. For strong disorder, the system scales to the normal Fermi-liquid fixed point (FFP) and the marginal effect disappears.

The properties of the MFFP are very different from that of the FFP. First of all, we find $\gamma_{2}^{*} \rightarrow-1$, implying that the spin susceptibility $\chi \rightarrow 0$ at the MFFP whereas $\chi \rightarrow \infty$ at the FFP. We also find $\gamma_{1}^{*}<0$. These behaviors can be interpreted in a Fermi-liquid phenomenology as showing that upon scaling, the effective interaction between quasiparticles becomes attractive $\left(\gamma_{1}^{*}<0\right)$, which results in suppression of local moments. Furthermore, we find that the effective magnitude of disorder $t$ is enhanced upon scaling $\left(t^{*}>t_{\text {initial }}\right.$ for arbitrary small $\left.t_{\text {initial }}\right)$. The enhancement of $t$ by interaction is absent in the normal Fermi liquid (to lowest order in $t$ ) and our result is in qualitative agreement with experimental observations that the effect of disorder is much enhanced in high- $T_{c}$. superconductors compared with normal metals. We have also examined numerically the scaling behaviors of $t$ in the intermediate regime $\left(t<t^{*}\right)$ and found that for all the in- 
put parameters we examined, $\gamma_{1}, \gamma_{2}, c_{n}$, and $C_{\kappa}$ (essentially when these parameters are of order 1), the intermediate scaling behavior can be fitted roughly by the form $t \sim \exp \left[\left(\omega_{c} / T\right)^{\delta}\right]$, where $\delta \sim 0.3$. This is again in qualitative agreement with experiments where the weaklocalization regime seems to be missing in high- $T_{c}$ superconductors. ${ }^{4,5}$ Unfortunately localization is not observed in our theory $\left(t^{*}\right.$ finite). However this is not surprising since the scaling equation we used is only valid to lowest order in $t$ and should not be applicable when $t$ becomes large $\left(t \geq c_{m}\right)$. It is also interesting to point out that the behavior $\chi \rightarrow 0$ seems to be observed in some of the high$T_{c}$ superconductors. ${ }^{2,17}$ Whether this is related to disorder is not clear at present. Notice that because of superconductivity, it is expected that the true ground state of the system should be a disordered superconductor which becomes an insulator if disorder is strong. However this possibility is not available in the present theory which suppresses the Cooper channel. Thus the theory is expected to be valid only for some $\xi<\xi_{s} \sim-\ln T_{c} \tau$, where $T_{c}$ is the transition temperature for formation of Cooper pairs. ${ }^{18}$ For $\xi>\xi_{s}$, the behavior of the system should be described by a dirty boson theory. ${ }^{18}$

For $t>t_{c}$, the system scales to the FFP where local moments appear and $\chi$ diverges. This result leads to the interesting possibility that there may be two kinds of insulating states for high- $T_{c}$ superconductors. For $t<t_{c}$, the system may be in a dirty boson state where "quasiparticles" are localized Cooper pairs which are spinless $(\chi \rightarrow 0)$ whereas for $t>t_{c}$, local moments start to form, resulting in diverging susceptibility. Unfortunately, the present lowest-order scaling theory cannot tell whether these possibilities truly exist.

In the Coulomb case, it is convenient to eliminate $\gamma_{1}$ by using the equality $1-2 \gamma_{s}=0$. It is then straightforward to show that no fixed point exists in the scaling equations with finite values of $\gamma_{2}^{*}, t^{*}$, and $Z^{*}$, and the only stable fixed point is the FFP $\gamma_{2}^{*} \rightarrow \infty$. Thus the marginalFermi-liquid effect can only show up as transient in the RG equations. The physical reason why no MFFP exists in the Coulomb case seems to be that the interaction between quasiparticles is always dominated by the Coulomb interaction $v(q)$ as $q \rightarrow 0$. The Coulomb interaction is repulsive whereas the MFFP needs attractive effective interactions. However, as in the short-ranged case, the present theory is expected to be valid only for some $\xi<\xi_{\text {s }}$ where $\xi_{s}$ is set by the transition temperature for formation of Cooper pairs. In particular, the behavior of the $\mathrm{RG}$ at $\xi<\xi_{s}$ may be dominated by the MFFP in which case the FFP is irrelevant. To test this possibility, we have solved the RG equations for the Coulomb case numerically for various input parameters $Z, \gamma_{2}, c_{m}$, and $t$ and find that if the ratio $t / c_{m}$ is small, the $\mathrm{RG}$ behavior is indeed dominated by the MFFP over most regions of $\xi<\xi_{c}$ where $\gamma_{1}$ and $\gamma_{2}$ become negative and $t$ increases with $\xi$. It is only when $\xi$ comes very close to $\xi_{c}$ that the FFP behaviors appear.

In summary, we have studied in this paper the effect of disorder on the normal-state properties of high- $T_{c}$ superconductors within a phenomenological marginal-Fermiliquid framework of diffusing quasiparticles. We find that the effect of disorder is enhanced by the so-called marginal-Fermi-liquid effect which is characteristic of the high- $T_{c}$ compounds, in qualitative agreement with experiments. ${ }^{4.5}$ We find a new fixed point for the RG equations which can be interpreted as describing a disordered Fermi liquid with attractive interaction. Both the conductance $\sigma$ and density of states $\rho_{1}$ are suppressed around this new fixed point, in contrast with the normal Fermi-liquid case where they are enhanced by interaction. The relevance of this new fixed point to experiments was discussed, where we point out that the new fixed point would probably be removed by a superconducting transition. We also want to emphasize that our theory is purely phenomenological. Though our results seem to compare favorably with experiments, justification of our approach depends on the structure of the final microscopic theory.

The authors thanks C. M. Varma for many illuminating discussions throughout the course of the work.
IJ. G. Bednorz and K. A. Muller, Z. Phys. B 64, 189 (1986).

${ }^{2}$ See, for example, Physical Properties of High Temperature Superconductors I and II, edited by D. Ginsberg (World Scientific, Singapore, 1990).

${ }^{3}$ See, for example, P. A. Lee and T. V. Tamakrishman, Rev. Mod. Phys. 57, 287 (1985).

${ }^{4}$ M. Z. Cieplak et al., Phys. Rev. B 39, 4220 (1989).

${ }^{5}$ See, for example, High Temperature Superconductivity, edited by K. S. Bell, D. Coffey, D. E. Meltzer, D. Pines, and J. R. Schrieffer (Addison-Wesley, Reading, MA, 1990).

${ }^{6}$ See, for example, C. DiCastro, in Anderson Localization, Proceedings of the International Symposium, Tokyo, Japan, edited by T. H. Fukuyama, Springer Proceedings in Physics Vol. 28 (Springer-Verlag, Berlin, 1989); G. Kotliar, ibid.

${ }^{7}$ G. Baskaran, Z. Zou, and P. W. Anderson, Solid State Commun. 63, 973 (1987).
${ }^{8}$ L. B. Ioffe and A. I. Larkin, Phys. Rev. B 39, 8988 (1989).

${ }^{9}$ C. M. Varma et al., Phys. Rev. Lett. 63, 1986 (1989).

${ }^{10} \mathrm{~K}$. Kotliar et al., Europhys. Lett. 15, 655 (1991).

I'C. Castellani, G. Kotliar, and P. A. Lee, Phys. Rev. Lett. 59, 323 (1987).

${ }^{12}$ A. M. Finkelstein, Zh. Eksp. Teor. Fiz. 84, 168 (1983) [Sov. Phys. JETP 5, 97 (1983)].

${ }^{13}$ C. Castellani et al., Phys. Rev. B 30, 527 (1984).

${ }^{14}$ D. Belitz and T. R. Kirkpatrick, Phys. Rev. Lett. 63, 1296 (1989).

${ }^{15}$ B. L. Altshuler and A. G. Aronov, Solid State Commun. 46, 429 (1983).

${ }^{16}$ G. Kotliar and C. M. Varma, Physica A 167, 288 (1990).

${ }^{17}$ J. Rossat-Mignod et al., Physica B 169, 58 (1991).

${ }^{18}$ T. K. Ng, Phys. Rev. B 43, 10204 (1991), and references therein. 